\title{
Fulminant myocarditis in children. Continuous renal replacement therapy to the rescue?
}

\author{
Patrick M. Honore ${ }^{1 *}$, Herbert D. Spapen ${ }^{2}$ \\ ${ }^{1} \mathrm{MD}$, PhD, FCCM, Professor of Intensive Care Medicine and Co-director of Research, ICU Department, Universitair Ziekenhuis Brussel, Vrije Universiteit Brussel, Brussels, Belgium \\ ${ }^{2} \mathrm{MD}$, PhD, FCCM, Professor of Intensive Care Medicine, Head of Unit and Director of Research, ICU Department, Universitair Ziekenhuis Brussel, Vrije Universiteit Brussel, Brussels, Belgium
}

Fulminant myocarditis (FM) is a devastating disease. Patients typically present with flu-like symptoms and then rapidly develop severe heart failure, cardiogenic shock and potentially fatal arrhythmias. ${ }^{1}$ In children, FM corresponds to $30-40 \%$ of cases of myocarditis with a mortality rate of up to $48 \%{ }^{2}$ Treatment is supportive, aiming to bridge the period of severe cardiac distress. ${ }^{1}$ In a recent issue of Revista da Associação Médica Brasileira, a novel and provocative treatment of FM is proposed based upon the intrinsic involvement of cardiac myosin. ${ }^{3}$ Extensive release of cardiac myosin is indeed thought to play a key role in the pathogenesis of FM, in particular by propagating autoimmune-induced myocardial inflammation and subsequent cardiac injury. ${ }^{4-6}$ Cardiac myosin is an hexamer consisting of two myosin heavy chains, each associated with two myosin light chains, i.c. an essential light chain (MLC-1) and a regulatory light chain (RLC). ${ }^{7}$ The $23-\mathrm{kD}$ MLC- 1 isoform has been identified as a sensitive biomarker of cardiac injury. ${ }^{8-10}$ Not surprisingly, the authors observed highly elevated MLC-1 levels in their patient cohort. ${ }^{4}$

More importantly, the authors focused on the removal of MLC- 1 from the bloodstream in an attempt to attenuate or suppress the noxious action of this molecule on the heart. This is supported by in vitro experience showing that RLC depletion reduced unloaded actin filament velocity and enhanced myosin-based isometric force approximately 2 -fold. ${ }^{8}$ Its relatively medium molecular weight makes MLC-1 particularly suitable for removal by convective continuous renal replacement therapy (CRRT).$^{11}$ Accordingly, a significant decrease of MLC-1 concentrations was noticed when continuous venovenous hemo(dia)filtration (CVVHDF) was applied. This brings to mind the seminal studies of Grootendorst et al., who observed a significant improvement in right ventricular ejection fraction and cardiac performance in endotoxemic pigs subjected to high-volume hemofiltration (HVHF) ${ }^{12}$ Infusion of ultrafiltrate from endotoxemic animals in healthy pigs caused a decrease in blood pressure and cardiac output suggesting that HVHF removed one or more vasoactive mediators responsible for sepsis-induced myocardial depression. ${ }^{13}$ However, a clinical benefit of HVHF on clinical course or outcome of human sepsis and septic shock has not been demonstrated. The current study also offers insufficient proof that lowering MLC-1 levels are associated with favourable clinical outcome. The reduction in MLC- 1 after a 48h CRRT session is certainly significant but CRRT alone cannot account for the observed $23 \%$ decrease. The "natural" metabolization rate of MLC-1 is unknown because a control group not receiving CRRT is lacking. An objective evaluation of left ventricular systolic function during treatment (e.g. with echocardiography) is missing. Moreover, the lower mortality rate in this small patient cohort should be weighed against baseline severity of disease and timing of the intervention. The CRRT approach also needs more in-depth evaluation before it can be recommended as a safe and effective adjunctive treatment for FM. Several factors may indeed determine substance removal during CRRT. CRRT-related issues comprise mode, volume, and dialysis membrane type and characteristics. Continuous venovenous hemofiltration $(\mathrm{CVVH})$ and $\mathrm{CVVHDF}$ are also different techniques. ${ }^{11}$ It is not clear to what proportion these epuration modes were used in the studied patients. Also, no information is provided regarding volume, intercurrent dialysis interruptions or on-off decisions, adverse events, and complications. Finally, it remains to 
be determined whether and to what extent MLC-1 is adsorbed on different membranes. Substance-related factors comprise volume of distribution, hydro- or lipophilic nature and protein binding. MLC- 1 is a hydrophilic molecule with a distribution volume of approximately $0.7 \mathrm{~L} / \mathrm{kg}$ and thus easily eliminated by convection. ${ }^{14}$ However, the light chain component of myosin expresses high affinity for ionized calcium which suggests a rather strong protein binding. ${ }^{15}$ The exact degree of protein binding is not known but may not exceed $80 \%$ to allow adequate CRRT removal. ${ }^{15}$ Elimination of MLC- 1 by CRRT is essentially determined by its molecular weight. Medium cut-off $(50 \mathrm{kD})$ membranes may therefore enhance MLC-1 removal without unwarranted loss of albumin. ${ }^{11}$ High cut-off $(60 \mathrm{kD})$ membranes may even perform better, but at the expense of more albumin depletion. ${ }^{16}$ Plasmafiltration which uses membranes with a cut-off value of approximately $1,000 \mathrm{kD}$ may be the most efficient option for adequate and swift MLC-1 removal. ${ }^{11,17}$

In conclusion, the authors presented a novel and provocative approach of FM in children. They identified MLC-1 as a prominent culprit of severe myocardial damage in FM and demonstrated substantial elimination of this molecule by CRRT. However, a potential link between lowering MLC-1 levels and improvement of myocardial inflammation or clinical outcome is poorly documented and speculative at the most. Nevertheless, further studies on the role of CRRT as adjuvant therapy for a disease with such grim prognosis must be encouraged. From a technical viewpoint, the use of CVVH with higher cut-off filters or plasmafiltration should be explored in more detail.

\section{AUTHORS' CONTRIBUTIONS}

$\mathrm{PMH} \&$ HDS designed the paper, participated in drafting the manuscript; and approved the final version of the manuscript.

\section{Conflict of interest}

The authors declare no conflict of interest.

\section{References}

1. Ginsberg F, Parrillo JE. Fulminant myocarditis. Crit Care Clin. 2013; 29(3):465-83

2. Saji T, Matsuura H, Hasegawa K, Nishikawa T, Yamamoto E, Ohki H, et al. Comparison of the clinical presentation, treatment, and outcome of fulminant and acute myocarditis in children. Circ J. 2012; 76(5):1222-8.

3. Sheng C, Zhang Z, Jia Y, Li Y. Changes in serum cardiac myosin light chain 1 levels in children with fulminant myocarditis during continuous blood purification. Rev Assoc Med Bras. 2017; 63(10):904-9.

4. Wang ZH, Liao YH, Dong JH, Li SL, Wang JP. Experimental study of autoimmune diseases, cardiac myosin-induced. Chinese J Cardiol. 2003; 31:937-40.

5. Cai G, Zhang J, Liu L, Shen Q. Successful treatment of experimental autoimmune myocarditis by adenovirus-mediated genetransfer of antisense CIITA. J Mol Cell Cardiol. 2005; 38(4):593-605.

6. Reddy J, Massilamany C, Buskiewicz I, Huber SA. Autoimmunity in viral myocarditis. Curr Opin Rheumatol. 2013 ; 25(4):502-8.

7. Yamashita H, Sugiura S, Fujita H, Yasuda Si, Nagai R, Saeki Y, et al. Myosin light chain isoforms modify force-generating ability of cardiac myosin by changing the kinetics of actin-myosin interaction. Cardiovasc Res. 2003; 60:580-8.

8. Pant K, Watt J, Greenberg M, Jones M, Szczesna-Cordary D, Moore JR. Removal of the cardiac myosin regulatory light chain increases isometric force production. FASEB J. 2009; 23(10):3571-80.

9. Celes MR, Malvestio LM, Suadicani SO, Prado CM, Figueiredo MJ, Campos EC, et al. Disruption of calcium homeostasis in cardiomyocytes underlies cardiac structural and functional changes in severe sepsis. PLoS One. 2013; 8(7):e68809.

10. Katus HA, Diederich KW, Ueller M, Remppis A, Schuler G, Kübler W. Myosin light chains release in acute myocardial infarction: non-invasive estimation of infarct size. Cardiovasc Res. 1988; 22(7):456-63.

11. Honore PM,Jacobs R, Joannes-Boyau O, De Regt J, De Waele E, van Gorp V, et al. Newly designed CRRT membranes for sepsis and SIRS--a pragmatic approach for bedside intensivists summarizing the more recent advances: a systematic structured review. ASAIO J. 2013; 59(2):99-106.

12. Grootendorst AF, van Bommel EF, van der Hoven B, van Leengoed LA, van Osta AL. High volume hemofiltration improves right ventricular function in endotoxin-induced shock in the pig. Intensive Care Med. 1992; 18(4):235-40.

13. Grootendorst AF, van Bommel EF, van Leengoed LA, van Zanten AR, Huipen $\mathrm{HJ}$, Groeneveld AB. Infusion of ultrafiltrate from endotoxemic pigs depresses myocardial performance in normal pigs. J Crit Care. 1993; 8(3):161-9.

14. Reetze-Bonorden P, Böhler J, Keller E. Drug dosage in patients during continuous renal replacement therapy. Pharmacokinetic and therapeutic considerations. Clin Pharmacokinet. 1993; 24(5):362-79.

15. Holroyde MJ, Potter JD, Solaro RJ. The calcium binding properties of phosphorylated and unphosphorylated cardiac and skeletal myosins. J Biol Chem. 1979; 254(14):6478-82.

16. Honoré PM, Jacobs R, Boer W, Joannes-Boyau O, De Regt J, De Waele E, et al. New insights regarding rationale, therapeutic target and dose of hemofiltration and hybrid therapies in septic acute kidney injury. Blood Purif. 2012; 33(1-3):44-51.

17. Cerdá J, Baldwin I, Honore PM, Villa G, Kellum JA, Ronco C; ADQI Consensus Group. Role of technology for the management of AKI in critically ill patients: from adoptive technology to precision continuous renal replacement therapy. Blood Purif. 2016; 42(3):248-65. 